\title{
Understanding the role of cholesterol in cellular biomechanics and regulation of vesicular trafficking: The power of imaging
}

\author{
Luciana de Oliveira Andrade \\ Departamento de Morfologia, Bloco J3, sala 310, Instituto de Ciências Biológicas, UFMG, Av. Antonio \\ Carlos, 6627, 31270-901, Belo Horizonte, MG, Brazil \\ E-mail: luoandrade@gmail.com
}

\begin{abstract}
Cholesterol is an important component of cell plasma membrane. Due to its chemical composition (long rigid hydrophobic chain and a small polar hydroxyl group), it fits most of its structure into the lipid bilayer, where its steroid rings are in close proximity and attracted to the hydrocarbon chains of neighboring lipids. This gives a condensing effect on the packing of lipids in cell membranes creating cholesterol-enriched regions called membrane rafts, which also congregate a lot of specific proteins. Membrane rafts have been shown to work as platforms involved with signaling in diverse cellular processes, such as immune regulation, cell cycle control, membrane trafficking and fusion events. A series of studies in the last two decades have linked many of these functions with the effects of membrane cholesterol content and rafts integrity on actin cytoskeleton organization, as well as its consequences in cellular biomechanics. This was possible by using microscopy techniques before and after manipulation of cholesterol content from cell plasma membrane, using agents that are able to sequester these molecules, such as cyclodextrins. In this review we'll give a personal perspective on these studies and how microscopy techniques were important to unravel the effects of cholesterol on actin and cellular mechanics. We will also discuss how actin and cholesterol contributes to control cell secretion and vesicular trafficking.
\end{abstract}

Keywords: Cholesterol, cell biomechanics, membrane trafficking, confocal microscopy, atomic force microscopy, laser tweezers, defocusing microscopy

\section{Introduction}

This year we are celebrating the 200th anniversary since Chevreul coined the term "cholesterine". Cholesterol is an animal sterol, a type of lipid and important component of cellular membranes. Its name derives from the ancient Greek, chole- (bile) and stereos (solid), due to the fact that it was first identified as a solid component of gallstones. Its first isolation in its crystalline form, from gallstones, occurred in 1768 by François Poulletier de la Salle. However it was only in August 1816 that Michel Eugéne Chevreul, a french chemist, identified and purified this crystalline substance from biliary calculi and named it as cholesterine [26]. Cholesterol is a hydrocarbon compound containing 27 carbon and 46 hydrogen atoms, plus 1 oxygen atom. It contains four hydrocarbon steroid rings in between the hydroxyl group and the hydrocarbon chain (Fig. 1). Most of its structure, due to its hydrocarbon composition, is hydrophobic. However, the presence of the hydroxyl group makes this molecule also partly hydrophilic giving it an amphiphilic property, characteristic of the lipid components of cellular membranes. The purpose of this review is to give an overview and a little bit of history of what is known today 


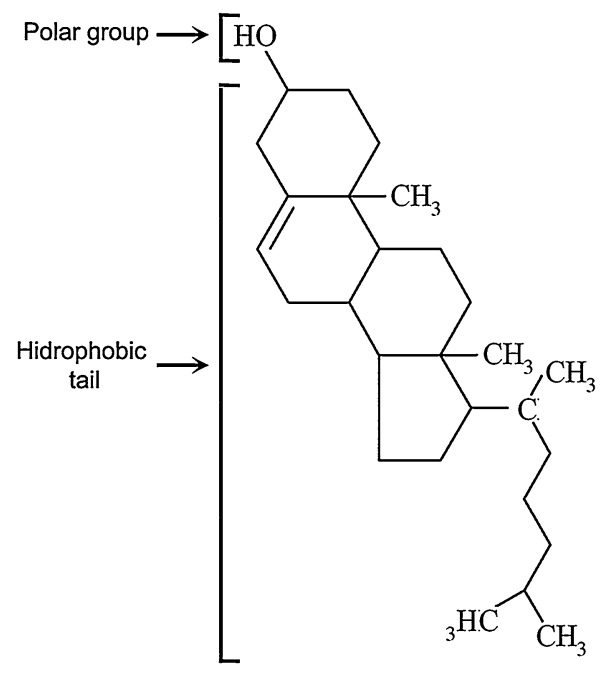

Fig. 1. Cholesterol chemical structure.

about cholesterol and membrane rafts and their effects on actin organization, cellular biomechanics and consequently membrane trafficking events.

\section{Cholesterol and membrane rafts}

Cholesterol displays a very important function as a component of cellular membranes, specially the cell plasma membrane where it is found in higher concentrations. Its positioning into the lipid bilayer and interaction with other lipids have a significant role in membrane fluidity together with other lipid components, such as the amount of sphingomyelin or the degree of saturation of the phospholipid acyl chains $[55,96,104,111]$. Cholesterol fits most of its structure into the lipid bilayer and only the small hydroxyl group faces the external environment. As a consequence, its steroid rings are in close proximity and attracted to the hydrocarbon chains of neighboring lipids. This gives a condensing effect on the packing of lipids in cell membranes [34]. However this effect seems to depend on the type of lipid it interacts with. As cholesterol hydrocarbon chain is rigid it tends to segregate together with fatty acids with saturated long acyl chains, especially sphingomyelin, leading to the formation of more compact liquid ordered and less fluid phases [88,91] (reviewed by [94]).

Cholesterol-enriched membrane regions, also called membrane rafts, were first identified as liquid ordered domains, containing high amounts of cholesterol and sphingomyelin and usually resistant to detergent solubilization (reason why they were first called detergent resistant membranes or DRMs). They were also known for its interaction with GPI-anchored proteins [94,113]. Despite its packaged structure, rafts were also shown to be dynamic structures, since their components still retained some lateral and rotational mobility [18]. In fact, one specific protein can display different partition coefficients with rafts, residing temporarily inside or outside these regions [85]. Detergent resistance was used as an important criterion to define these domains. However, it has been shown that other characteristics are important to designate a membrane raft [69]. Therefore, after a Keystone Symposium on Lipid Rafts and Cell function, a set of parameters is now used to determine the so-called membrane rafts region. The membrane rafts are identified as small heterogeneous and dynamic membrane domains, of approximately 10-200 nm, which are capable of compartmentalizing specific cell processes [83]. 
Membrane rafts, due to its protein and lipid composition, compartmentalize a lot of important cell functions [92]. They have been shown to work as platforms involved with signaling in diverse cellular processes, such as immune regulation, cytoskeleton reorganization, cell cycle control, as well as membrane trafficking and fusion events, among others $[27,70,95,105,109]$. The association or dissociation of proteins and receptors with rafts could regulate many of these functions. Rafts might then be seen as platforms that concentrate receptors in a new microenvironment, whose functions are protected from non-raft enzymes, as is the case for phosphatases that could affect signaling processes [93].

\subsection{Cyclodextrins as a tool to study membrane rafts}

One way to indirectly study cholesterol enriched membrane microdomains is to sequester cholesterol from cell membranes using cyclodextrins. Cyclodextrins are ring like structures made of six to eight glucopyranose units, bound via -1,4- glycosidic linkages [62]. The advantage of this kind of molecules is that they have mainly a hydrophobic interior, while its exterior is hydrophilic, giving it the ability to carry and "solubilize" hydrophobic molecules in an aqueous environment [84]. Cyclodextrins have been widely used as drug carriers. Their affinity to different hydrophobic molecules is defined by the size of their rings. Cyclodextrins are divided into three different compounds, differing on the number of glucopyranose units forming the ring: the alpha $-\alpha$ (containing 6 glucopyranose units), beta $-\beta$ (containing 7 glucopyranose units) and gamma $-\gamma$ (containing 8 glucopyranose units) [80] (Fig. 2A). Since it is a ring structure, it can fit molecules in its interior. Affinity for the ring interior will depend not only on its hydrophobicity, but also its size (Fig. 2B). The $\beta$-cyclodextrins are the ones that are usually used as carriers and are also the ones that have the highest affinity to cholesterol [80], especially the methyl modified $\beta$-cyclodextrin [58,110]. Since plasma membrane is usually enriched in cholesterol [65] and cyclodextrins have low permeability through cellular membranes, most of the cholesterol sequestered by this drug comes from cell plasma membrane [31]. Additionally, it has been shown that cholesterol sequestration by $\mathrm{M} \beta \mathrm{CD}$ occurs biexponentially, which indicates the existence of two kinetic pools of cholesterol efflux, a fast and a slow pool [110]. Therefore it has been stated that most likely these two independent kinetic pools come from sequestration of cholesterol-enriched domains, rafts (fast pool), and the rest of membrane cholesterol (slow pool). Therefore use of $\mathrm{M} \beta \mathrm{CD}$ can be a valuable tool to study cell processes related to membrane rafts.

\section{Cholesterol and actin cytoskeleton organization: Imaging cells using confocal microscopy}

Altering the levels of cholesterol in cellular membranes will interfere with rafts organization. Decrease in membrane cholesterol content, for example, leads to rafts disruption and consequently alters, directly or indirectly, the cellular processes linked to these regions, such as signaling, membrane trafficking and cytoskeleton organization. Cytoskeleton organization, in particular, seems to play an important role in rafts cellular functions. It has long been shown that membrane rafts are not only enriched in signal transduction molecules, but also actin and actin binding proteins [47,75]. Additionally, it was demonstrated that changes in cytoskeleton organization upon rafts disruption also alters signaling processes linked to this platform $[54,73]$.

One simple way to show cytoskeleton organization is to image these structures using fluorescence microscopy, especially Confocal Laser Scanning Microscopy (CLSM or just Confocal Microscopy $\mathrm{CM}$ ). Advantage of confocal microscopy over regular epifluorescence microscopy is the fact that it collects images into specific focal plans (optical sections), without the interference of out of focus light, 
A

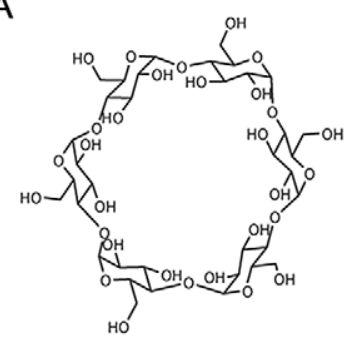

$\alpha-C D$

B

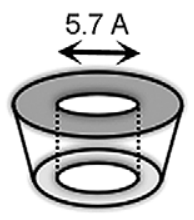

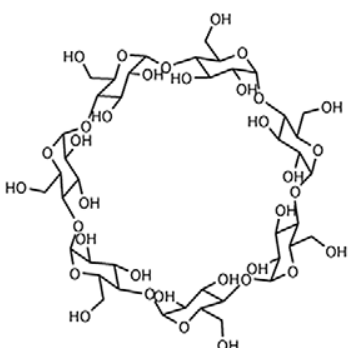

$\beta-C D$

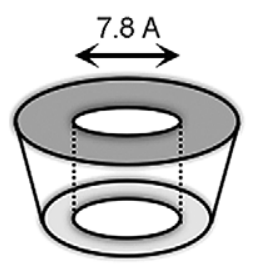

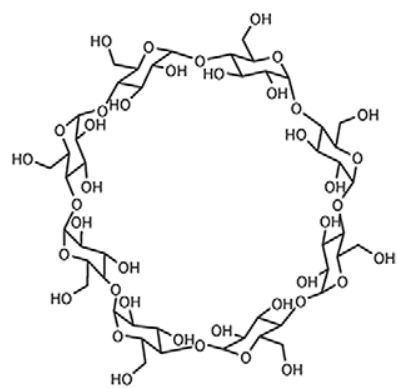

$\gamma-C D$

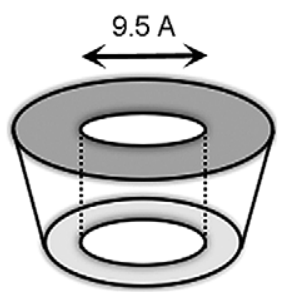

Fig. 2. (A) Chemical structures of the three different types of cyclodextrin, alpha $(\alpha)$ - containing 6 glucopyranose units, beta $(\beta)$ - containing 7 glucopyranose units and gamma $(\gamma)$ - containing 8 glucopyranose units. (B) Representative images of the rings formed by the three different cycodextrins and the respective size of their pockets.

by scanning a sample point by point and assembling each pixel information to form an image. It therefore allows three-dimensional reconstructions of high quality images. Imaging actin filaments (f-actin) organization was made quite easy using this kind of technique with the aid of fluorophore-conjugated phalloidin. Phalloidin is a toxin isolated from the death cap mushroom (Amanita phalloides), which was found to selectively bind and stabilize f-actin [67]. It does so by binding f-actin in between subunits, holding them together, [9] (Fig. 3A). Since fluorescent conjugated phalloidin still retain their ability to bind f-actin, it became a very important tool to study organization of these filaments in cells [108].

The association of actin with membrane regions enriched in cholesterol was known for a long time [47, $48,75,111]$. However, one of the first demonstrations that rafts disruption by cholesterol sequestration could have an effect in actin organization came from a work from Kwik and co workers in 2003 [63]. They showed that the role of rafts in controlling cell actin cytoskeleton organization was linked to the association of cholesterol enriched domains with phosphatidylinositol 4,5-bisphosphate [PI(4,5)P2] and its role in actin polymerization [23,112]. Kwik and co-workers showed that disruption of membrane rafts in fibroblast by cholesterol sequestration led to the dispersion of [PI(4,5)P2] and consequently the induction of actin polymerization foci [63]. Imaging Rhodamine-Pahlloidin labeled f-actin in control and $\mathrm{M} \beta \mathrm{CD}$ treated cells using $\mathrm{CM}$, they observed that cholesterol sequestration from cell plasma membrane led to a decrease in the number of stress fibers, especially thinner stress fibers in cells cytoplasm. They have also observed small punctate phalloidin staining in $\mathrm{M} \beta \mathrm{CD}$ treated cells, indicating the increase in actin polymerization sites. The increase in actin polymerization was corroborated by CM images showing less gelsolin staining in cholesterol-depleted cells. Later, Klausen and colleagues, studying the effects of cholesterol depletion in Ehrlich-Lettre ascites (ELA) cells, also showed that cholesterol depletion interfered with actin organization [59]. However, in this work it was shown that cholesterol sequestration led to an increase in cortical actin cytoskeleton with a significant augment in stress fibers 
A

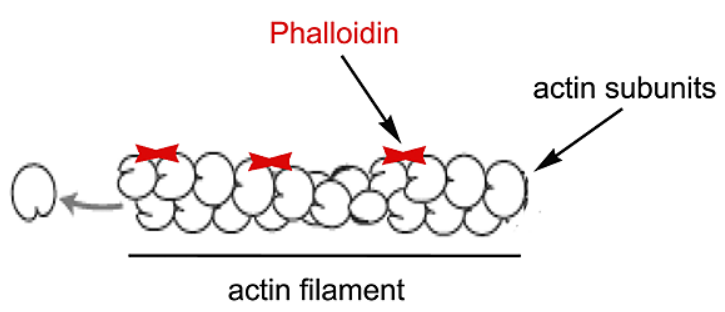

B

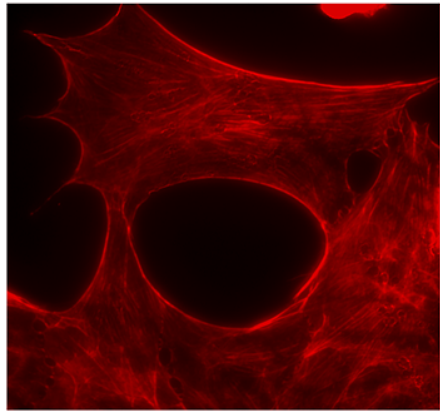

Control

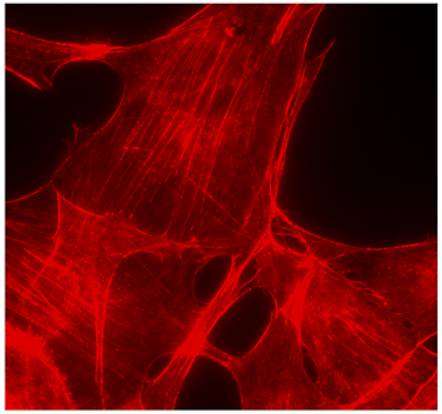

M $\beta C D$

Fig. 3. (A) Representative image of actin filaments and the sites of binding of phalloidin. (B) Fluorescence images of mouse embryonic fibroblasts treated or not (control) with $\mathrm{M} \beta \mathrm{CD} 10 \mathrm{mM}$, fixed with $4 \%$ paraphormadehyde and labeled with phalloiding conjugated with Alexa fluor 546 (Invitrogen ${ }^{\circledR}$ ). Arrows indicate the actin stress fibers in $\mathrm{M} \beta \mathrm{CD}$ treated cells.

leading to alterations in volume-regulated anion current in these cells. They also showed that change in actin cytoskeleton organization occurred not due to alterations in [PI(4,5)P2], but due to activation of Rho, a known regulator of actin polymerization. Subsequent work from Qi and co-workers, studying an osteoblast cell lineage (MC3T3), showed that cholesterol regulation of actin cytoskeleton involves not only Rho, but also ROCK (Rho Kinase), Src kinase and Cav-1 (caveolin-1) [87]. They not only observed extreme re-organization of the actin cytoskeleton with an increase in stress fiber formation, but they were able to show that these effects of cholesterol were due to the activation of two pathways linked to src activation: RhoA and ROCK activation, as well as Cav-1 phosphorlation. Later, our group also showed that cholesterol sequestration induced stress fiber formation in neonatal murine primary cardiomyocytes (CM) [50]. Figure 3B shows some representative images of fibroblasts treated or not with $\mathrm{M} \beta \mathrm{CD}$ and the effects of the treatment on actin cytoskeleton organization. In this study we were able to demonstrate that for these cells, as well as for ELA and MC3T3, stress fiber formation was due to RhoA activation. Additionally, and most important, in a follow up work we were able to show that cholesterol sequestration was able to not only reorganize pre-formed f-actin, inducing the formation of stress fibers, but also to induce de novo polymerization of actin [51]. These experiments were performed by treating cells with latrunculin, a potent drug able to sequester actin monomers leading to complete actin filaments destabilization, previous to treatment with $\mathrm{M} \beta \mathrm{CD}$.

A lot of other work corroborated these data showing that cholesterol depletion from cell plasma membrane leads to actin polymerization and reorganization. Most importantly, many of these works showed that changes in the actin cytoskeleton induced cell stiffness and changes in biomechanical properties of cells $[27,29,51,56,78,97]$. 
Although all these works were performed using a drug, $\mathrm{M} \beta \mathrm{CD}$, which could be considered nonphysiological, the results could certainly reflect many pathological situations where plasma membrane cholesterol content is affected, such as in many lipid disorders. Mouse models of hypercholesterolemy, with high levels of LDL (Low density Lipoprotein), one of the major causes of atherosclerosis in humans, seems to cause the same differences in the levels of cholesterol induced by $\mathrm{M} \beta \mathrm{CD}$ treatment $[37,87]$. In fact, oxLDL was shown to be capable of sequestering cholesterol from cell plasma membrane [14, 61]. Then a series of studies were published showing that cell exposure, specifically endothelial cells, to the oxidized form of LDL (oxLDL) particles also led to reorganization of the actin cytoskeleton. LDL particles are complex structures formed of different lipids and the proteins ApoB [49]. Oxidation of these particles leads to changes such as loss of antioxidant molecules, formation of shiff's base in aldehydes from ApoB protein and production of oxysterols, peroxides and others. OxLDL, according to Parthasarathy et al. (2010), can be defined as: a particle derived from circulating LDL that may have peroxides or their degradation products generated within the LDL molecule or elsewhere in the body associated with the particle [81]. The excess LDL in the blood results in its accumulation in the intima of arteries leading to its oxidation. This excess of oxLDL has been implicated in a series of process that leads to atherosclerosis, such as macrophage recruitment/activation and endothelial cell dysfunction. While much is known about the effects of oxLDL in macrophages little is known about their effect on endothelial cells and how it leads to its dysfunction, thus the interest in understanding the effects of oxLDL on actin organization in these cells. In parallel to some of the studies performed with $\mathrm{M} \beta \mathrm{CD}$ it was demonstrated that cell exposure to oxLDL, as well, leads to cortical cytoskeleton reorganization, changing the mechanical properties of these cells $[21,28,60,68]$.

\section{The study of cellular biomechanics in cholesterol depleted cells}

As mentioned, the increase in actin stabilization at cell periphery and stress fiber formation leads to changes in cellular biomechanics. Cell actin organization, and consequently cell mechanics, is recognized to be a major player in various cell responses to internal and external environment $[33,43,45,66,106]$, therefore the interest in studying the effects of plasma membrane cholesterol levels and rafts organization in cellular mechanics. A pioneer work in this field was published by Byfield and co-workers, working with aortic endothelial cells, where they showed that plasma membrane cholesterol content do relate with levels of membrane stiffness [20]. After this, a lot of other papers were published. Most of them used microscopy techniques to study the biomechanical effects of cholesterol depletion induction of stress fiber formation. Below I will give a brief description of some of these techniques and the results obtained with them.

\subsection{Use of atomic force mycroscopy}

Atomic Force Microscopy (AFM) is based on a scanning probe microscopy, which is designed to measure local properties such as height and friction with the use of a probe. AFM was first described in 1986, by Binnig and colleagues [12], and is the result of the combination of two types of microscopy, Scanning Tunneling Microscopy [13] and Stylus Profilometer [44]. By combining these two techniques, it is possible to resolve surface and force at the atomic level. AFMs work by measuring the force between a probe (a sharp tip) and a sample. The tip scans through an object to obtain information of its surface. The force on the tip is kept small and constant. It also has a feedback mechanism to be able to trace over individual atoms without damaging the sample. Probes used in AFM are pyramidal in shape and 
usually have less than $10 \mathrm{~nm}$ in radius [42]. The tip is attached to a spring in the form of a cantilever and the attractive and repulsive forces between the tip and the sample are measured by the deflections of the cantilever $[42,46]$. This force on the tip can also be used to form a tridimensional image of the surface of the sample. The deflection of the cantilever is measured using a laser, which is reflected from the cantilever and detected by a quadrant photodiode [42]. Because it can image non conducting substances it was possible to use this kind of technique in biological samples. Since its development until now, much has improved in this type of microscopy for biological uses. Newest generations of AFM, destined to biological studies, are now combined with other microscopy platforms, such as epifluorescence, to perform live cell imaging and improve data analysis. AFM is then able to provide, with high resolution, cell topography at the nanometer level, as well as to measure physical properties, such as viscoelasticity, stiffness and adhesion, which also provides information about cell cytoskeleton organization and cell organelles. [42]. To measure mechanical properties using AFM, a force is applied to the tip onto the sample and then the deformability caused by this force is evaluated. For this, vertical displacement of the cantilever, as well as its deflection, is measured and then converted to force-versus-displacement curves, or just force curves [42]. Tips for optimal conditions in measuring cellular mechanics can de found in a recent publication from Gavara (2016) [42].

Byfield and co-workers had previously demonstrated that cholesterol sequestration leads to increased membrane cell stiffness by measuring the length of membrane deformation aspirated into a glass micropipette [20]. Although likely, due to technical limitations, this study could not prove that these effects were in fact a consequence of actin rearrangements near cell plasma membrane. Later, Sun and co-workers tested this hypothesis by using AFM to study membrane cytoskeleton adhesion in cells subjected to cholesterol sequestration from cell plasma membrane and their effects on cellular biomechanics [97]. Through this technique he and colleagues assessed the biophysical properties in control, non-treated, bovine aortic endothelial cells (BAECs), as well as BAECs after treatment with $\mathrm{M} \beta \mathrm{CD}$ for 30 minutes. AFM was performed using tether-pulling experiments to mimic the collision of leukocytes with endothelial cells during blood flow, which may result in tether formation. They measured the force to pull a tether from the membrane at a constant velocity and analyzed the dependence of force on this velocity $[17,53]$. They then evaluated surface viscosity, which associates with not only the lipid bilayer, but also the underlying cytoskeleton [53]. Their results have shown that cholesterol sequestration does increase tether-pulling forces and leads to increased cell stiffness. They have also tested BAECs that have been exposed to $\mathrm{M} \beta \mathrm{CD}$-cholesterol complexes in order to donate and enrich their membrane with cholesterol. The results obtained with these cells were the opposite of that obtained with cells presenting reduced cholesterol content. These data not only confirmed the role of cholesterol in cytoskeleton organization, but also its influence in cell biomechanical properties. Later, Chouinard and co-workers using AFM imaging and force measurements showed that treatment of endothelial cells with oxLDL also led to increase in cell stiffness [28]. They performed the experiments in the course of 24 hours and analyzed the elastic parameters of cells after 30 and 120 minutes or 6,12 and $24 \mathrm{~h}$. AFM force measurements showed an increase in cell stiffness after $24 \mathrm{~h}$ treatment with oxDL, but not LDL. These results were in agreement with previous results obtained by Byfield et al. (2007), which evaluated cell membrane deformation using micropipette aspiration [21].

\subsection{Use of optical tweezers}

Optical tweezers (OT) is a technique in which a high focused laser beam is used to provide an attractive or repulsive force to physically hold and move microscopic dielectric objects similar to tweezers. Ashkin 
and colleagues in 1986 provided the first experimental demonstration of the use of single-beam gradient force radiation pressure to trap particles [5]. By using a strongly focused beam the axial gradient force was so large that it dominated the axial stability allowing to efficiently trap particles. Using this technique they were able to trap dielectric particles in the size range from $10 \mu \mathrm{m}$ down to $25 \mathrm{~nm}$. In 1987, Askin and Dziedzic demonstrated its first application in biology, by trapping viruses and bacteria in aqueous solution [7]. Later, after modification of the laser beams to infrared ones, they were able to use it to trap single cells and to pull tethers from cell membranes [7]. It was also used in biology to trap and measure forces of single motor molecules and mechano-enzymes [6,15,98]. But it wasn't until 1995 that OT was used to study the first mechanical parameters in cells by pulling membrane tethers with the use of beads trapped by OT [32]. Studying the growth cone membranes of neuronal cells they were able to measure the force involved to pull a membrane tether and therefore determine mechanical parameters involved in this process. For this they used a system to track the position of the bead during the tether formation to calculate the force to pull the tether. Also, by calculating the tether radius they were also able to measure membrane viscosity [32].

One of the first uses of optical tweezers to address alterations in mechanical properties of cells, due to changes in cholesterol levels at the plasma membrane, was made by Kwik and co-workers in 2003 [63]. They demonstrated that decrease in cholesterol levels at the plasma membrane, besides altering actin cytoskeleton organization, also decreased protein lateral mobility. They used gold beads labeled HLA molecules to allow them to be trapped by the laser tweezers and then to be moved along the plane of the cell membrane and placed about $1 \mu \mathrm{m}$ from the original location. While in non-treated cells HLA molecules remained for a while in the place where it had been moved to, in $\mathrm{M} \beta \mathrm{CD}$ treated cells, the HLA molecules returned right back to its first location, indicating they were anchored to cell cytoskeleton. This hypothesis was corroborated by the work performed by Sun and colleagues, using FRAP (Fuorescence recovery after Photobleaching) showing that the effect of cholesterol sequestarion on protein mobility could be abrogated by further treatment with latrunculin, a drug capable of disrupting actin filaments, indicating that membrane cell stiffness was also responsible for the effects on protein diffusibility along the membrane [97]. Later, Khatibzadeh et al. (2012) used the optical tweezers to pull a tether from plasma membranes of control, $\mathrm{M} \beta \mathrm{CD}$ or $\mathrm{M} \beta \mathrm{CD}$-cholesterol treated cells. It did so by optically trapping $4 \mu \mathrm{m}$ fluorescent polystyrene beads and using them to attach and pull tethers from cell membranes [56]. Optically measuring the displacement of the bead in relation to the trapping center they were able to calculate the force used to pull the tether. In $\mathrm{M} \beta \mathrm{CD}$ treated cells tether pulling forces were more than twice the force necessary to pull a tether from control cells [56]. On the other hand, cells treated with $\mathrm{M} \beta \mathrm{CD}$-cholesterol, in order to enrich cell membranes with cholesterol, led to a decrease in this tether pulling force. These results indicated that interaction of the actin cortical cytoskeleton was stronger with membranes containing lower amount of cholesterol. A follow up work from the same group, now also using cells treated with latrunculin, confirmed previous results showing that cholesterol disruption alters membrane interaction with the actin cytoskeleton leading to increased cell stiffness [57]. These results also confirmed that both plasma membrane and cytoskeleton contribute to the viscoelastic behavior observed for the membrane tethers. Additionally and most important, they showed that the effects of plasma membrane itself in mechanical properties of cells are relatively minor in comparison with the effects of the cytoskeleton. On the other hand, changes in plasma membrane content, as in the case of cholesterol, might induce changes in the cytoskeleton and therefore indirectly contribute to cellular mechanical properties [57]. These results were corroborated by a work from our group, showing that the levels of the plasma membrane cholesterol content influence cell rigidity [51]. Using optical tweezers to pull membrane tethers we were also able to demonstrate that membrane cholesterol sequestration via 
$\mathrm{M} \beta \mathrm{CD}$ treatment leads to increase in tether pulling forces compared to non-treated cells. By measuring the tethers radii using Scanning Electron Microscopy we were also able to measure surface tension and bending modulus and both were increased in cells with decreased cholesterol content [51]. In this work we suggested that this increase in cell rigidity and surface tension might be linked to the fact that, before cholesterol sequestration, actin-binding proteins are clustered within membrane rafts at specific locations in the membrane. Upon cholesterol removal and membrane rafts disruption, actin-binding proteins also disperse along the plasma membrane forming more connections between membrane and cytoskeleton at the entire cellular surface. Protein dispersion and redistribution after membrane cholesterol content is decreased has been previously reported supporting this hypothesis [70,102].

\subsection{Use of defocusing microscopy}

Agero and colleagues first described the use of Defocusing microscopy to measure alterations and curvatures in membrane surfaces of cells in 2003, while working at the laboratory of Dr. Osacr Nassif Mesquita at Federal University of Minas Gerais (UFMG - Brazil). Defocusing Microscopy consists of introducing defocalization of a phase-transparent object (like adhered cells) in an optical microscope in order to be able to visualize it [1]. In this situation any irregularities seen in its surface can be detected through the image contrast observed. Defocusing had been used earlier with laser light to image the spatial and temporal fluctuations of optical microstructures of seawater. In this study by Agero et al. (2003) they used the defocusing theory to image surface curvature fluctuations of resting macrophages and during single phagocytosis events. They demonstrated in their study that the image contrast was proportional to the two-dimensional of the Laplacian of the phase difference introduced by the phase object. Therefore all the information obtained from the contrast was directly related to the information of membrane curvature. Membrane curvature in turn is a consequence of actin cortical cytoskeleton reorganization and could be used to determine these alterations. In these experiments they imaged macrophages for 2 hours and determined the spatial and temporal contrast correlations, which gave the relaxation time. Temporal correlation was obtained from the contrast of every pixel in different times, averaged over several pixels of a chosen region and the spatial correlation was obtained from the simultaneous value of the contrast in different pixels, where the average is done over many video frames. Later they used this parameters collected via DM to measure mechanical properties of the cell, such as the relaxation time and amplitude of membrane fluctuations [2,30].

Our group, in collaboration with the group of Dr. Mesquita and Dr. Agero, was able to apply DM to measure the biomechanical properties of fibroblasts treated or not with $\mathrm{M} \beta \mathrm{CD}$. The advantage of using this method was the fact that we could temporarily follow the changes in cell membrane surfaces and biochemical properties of the cells along the cholesterol sequestering process. In these experiments cells were imaged 10 minutes before the addition of the drug and were followed for as long as forty-five minutes after [51]. The data was fitted using a temporal autocorrelation function, as described earlier $[1,30]$ and the relaxation time and amplitude of curvature were determined. We observed that the relaxation time significantly increased upon cholesterol sequestration, especially between 10-30 minutes after drug administration. However we also observed that relaxation time returned to lower levels in the last 5 minutes of the experiment. We also evaluated the amplitude of membrane curvatures and showed that membrane fluctuations, as expected, were less intense in membranes with a lower content of cholesterol. These data corroborated all the other data from the literature and from our group, showing that cholesterol sequestration leads to cell stiffness. These experiments also allowed us to show that the effects of cholesterol sequestration from cell plasma membrane in cellular mechanical properties occur very fast, 
after the first 10 minutes of $\mathrm{M} \beta \mathrm{CD}$ addition and peaks between 20 and 30 minutes after addition of the drug.

\section{Effects of membrane cholesterol on cell secretion}

It has been demonstrated that SNARE proteins, a large group of small membrane proteins essential for vesicle fusion events, are associated with rafts [64,70,79]. SNAREs are divided into two groups: the v-SNAREs, localized at the vesicles (vesicle-associated membrane protein - VAMP/synaptobrevin family), and the t-SNAREs, localized at the target membranes (syntaxin family and SNAP-23 family). During vesicle fusion to the target membrane, one v-SNARE associates with two t-SNAREs to form a ternary complex responsible for bringing the two membranes closely together allowing them to fuse [25]. Therefore it is imperative that the two t-SNARES are clustered together at the moment of vesicle fusion in order to form the ternary complex. Many groups have shown not only the presence of one or more SNAREs inside rafts, but also that the presence or reallocation of these proteins in or outside rafts may interfere with vesicle fusion events and consequently exocytosis, especially in regulated cell secretion $[24,64,79,102]$. In some cases, the clustering of SANREs into rafts seem to facilitate vesicle fusion and exocytosis, as it is the case for mast cells [86]. Mast cell activation, via $\operatorname{IgE}$ binding to its receptors, leads to rearrangement of SNARE proteins into rafts regions. This seems, at least in part, to account for regulated secretion of mast granules [86]. The same was observed for sperm capacitation, a process involved with preparation for acrosome fusion during egg fertilization [16,41]. During capacitation, SNARE proteins are recruited and clustered into rafts allowing the fusion of acrosomes with the apical region of sperm head membranes. Therefore, since rafts disruption will lead to protein dispersion, it could also compromise these membrane fusion events. In fact, this was the case for secretion of $\beta$ pancreatic cells and neuroendocrine cells. Cholesterol sequestration led to inhibition of cell secretion [24,64]. However, it was also observed that cholesterol depletion from axons of crayfish neuromuscular junctions, despite inhibiting evoked synaptic transmission, led to an increase in spontaneous quanta transmitter release [114]. These results suggested that cholesterol sequestration and consequent rafts disruption could also lead to unregulated secretion events. This hypothesis was corroborated by the fact that synaptic vesicle fusion during induced exocytosis is an event regulated and dependent on calcium, while these spontaneous events were not [114].

As previously mentioned in the beginning of this review I was very much interested in understanding the mechanisms underlying the cholesterol influence on $T$. cruzi-host cell invasion. Two independent works had shown that parasite invasion is compromised when host cell membrane cholesterol was sequestered using $\mathrm{M} \beta \mathrm{CD}[10,38]$. However no mechanism for this phenotype was presented. It was recently shown that $T$. cruzi subverts the plasma membrane repair mechanism from eukaryotic cells to gain entry into the host cell cytoplasm $[39,40,99]$. When this parasite interacts with host cells it can either induce intracellular calcium signaling events through host cell surface receptor activation $[19,36,101,107]$ or small microinjuries induced by parasite friction at the host cell surface [39]. Both events lead to calcium increase due to its release from intracellular stocks or through its influx from the extracellular milieu [100,101]. The high levels of free cytoplasmic calcium binds to Synatotagmin VII (SytVII), a calcium sensor present in lysosomes, which then helps the SNAREs ternary complex formation that will culminate with the fusion of lysosomes with plasma membrane $[22,89,90]$. Lysosome exocytosis is then followed by compensatory endocytosis, such as occurs in plasma membrane repair, and the latter drives 
parasite into the cell [39]. Lysosome fusion was also shown to be imperative for entry and maintenance of the parasites inside host cells [3]. We first thought that cholesterol sequestration, and consequently rafts disruption, was compromising lysosome fusion with host cell plasma membrane, such as previously reported for other secretory vesicles, and thus decreasing parasite invasion. However we identified that membrane cholesterol disruption was, on the contrary, inducing lysosome fusion events [50]. The lysosomal exocytic events triggered by cholesterol sequestration were independent of calcium or the lysosomal calcium sensor, Syt-VII, indicating they were unregulated events, such as the ones observed by Zamir and collegaues in cholesterol depleted axons from crayfish neuromuscular junctions [114]. Another interesting aspect was that it occurred specifically from a pool of lysosomes localized closer to the cell plasma membrane, which we later identified as a pool recruited during parasite-host cell invasion [50,52].

At this point one question still remained. What was driving lysosomal exocytosis in the absence of calcium? It was possible that reallocation of SNAREs would allow them to randomly cluster and possibly trigger this events. However, since the secretion occurred independently of calcium, something else would still have to help triggering the fusion. Although cortical actin was always seen as barrier for cell secretion, later was shown that it could also help in this process $[8,72,74,82]$. In 2001, Pedleton and co-workers showed that complete abrogation of actin cytoskeleton using latrunculin inhibited mast cell secretion, indicating that actin filaments had a positive role in cell secretion [82]. In $\beta$-cells from pancreas it was shown that actin coats secretory vesicles after fusion, working in the regulation of post fusion events [76,103]. It was proposed that the coating of secretory vesicles with actin is able to stabilize vesicle-target membrane fusion aiding in this process. A lot of other subsequent studies showed that actin and myosin participated in these post fusion events, controlling the time of pore openness $[4,11,35]$. Also, Masedunskas and co-workers showed that, for large secretory vesicles from $\beta$-adrenergic cells, actin and Myosin participate not only in post fusion events but also in the actual fusion process [71]. In this work the authors used intravital microscopy to show that actin associated with myosin II participate in the fusion process by promoting the full fusion of vesicles [71]. In the same year, Nightingale showed that actin participated in different processes during the secretion of Weibel-Palade bodies (WPBs), regulated secretory organelles of endothelial cells [77]. First it was responsible for anchoring WPBs to control their premature fusion and secretion. After cell stimulation, dynamic actin filaments together with myosin were seen at the bottom and around WPBs, concomitantly with granule secretion. This indicated that actin would exert a force at the granule to help the release of its content [77]. In the light of these works, it seemed plausible that lysosomes exocytosis, induced upon cholesterol sequestration from plasma membrane, could be also triggered by cell cytoskeleton reorganization. In our work we showed that cholesterol sequestration from plasma membrane led to the exocytosis of a specific pool of lysosomes localized at the cell cortex, as seen by lysosomal cell distribution before and after treatment (lysosomal dispersion assay) [50]. This could represent docked lysosomes in which actin dynamics, induced by cholesterol sequestration, could be working to push these organelles into closer contact with plasma membrane activating the exocytic machinery (Fig. 4). Later work from our group corroborated this hypothesis [51]. First, we showed that cholesterol sequestration induces actin rearrangements, through Rho A activation, which could be contributing to form the dynamic actin filaments that were previously shown to form the rings responsible to push and help vesicle secretion [77]. Second, although treatment with latrunculin-A also led to lysosome secretion, it preferentially induced the secretion of a more internal pool. This could be explained by the fact that actin, as previously shown, can work as a barrier controlling the premature fusion of internal vesicles [8,74]. Without this barrier, 
A

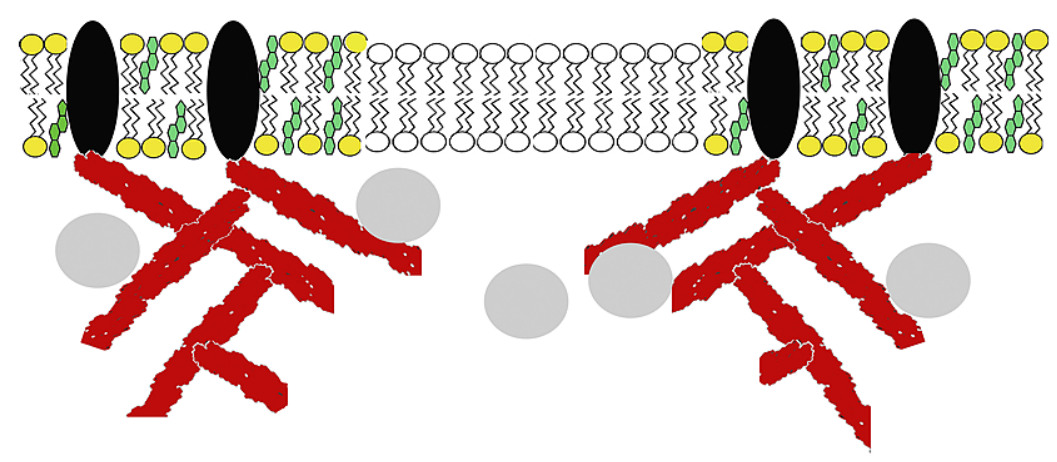

B

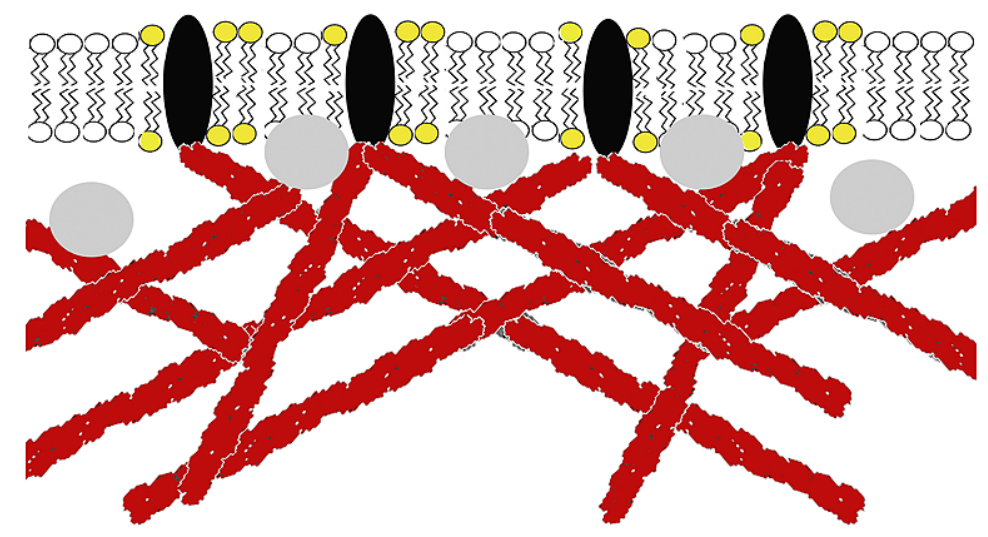

Fig. 4. Model of exocytosis triggered by cholesterol sequestration from plasma membrane. (A) Plasma membrane containing cholesterol and presence formation of the membrane rafts microdomains. In these domains, actin-binding proteins hold actin filaments in specific locations. Peripheral lysosomes are docked to these actin filaments. (B) Plasma membrane after cholesterol sequestration. Actin-binding proteins reallocate along the membrane due to the disruption of the membrane rafts. Actin filaments also increase in size due to actin polymerization induced by cholesterol sequestration. In this scenario, reorganized actin filaments push peripheral lysosomes closer to the cell plasma membrane secretion occurs.

the internal pool of lysosomes, which could represent a different pool from the ones usually docked at the cell cortex, are free to get to the cell plasma membrane and fuse. Corroborating this idea, treatment of cells with latruculin-A followed by treatment with $\mathrm{M} \beta \mathrm{CD}$ leads to higher levels of lysosomal secreted enzymes, when compared to either treatment separately [51]. Additionally, lysosomal dispersion assays of cells, treated with $\mathrm{M} \beta \mathrm{CD}$ alone or with latrunculin-A followed by $\mathrm{M} \beta \mathrm{CD}$ treatment, show different patterns. While in the $\mathrm{M} \beta \mathrm{CD}$ treated cells we observed only the loss of peripheral lysosomes, for the ones treated with latrunculin- $\mathrm{A}+\mathrm{M} \beta \mathrm{CD}$ we observed also a significant loss of more internal lysosomes as well [51]. These data brings new insight in the role of actin in lysosomal secretion, indicating that it not only participates in lysosome secretion, but also may help subdivide pools involved with different processes in the cell. This may also be the case for other secretory vesicles and further work need to be done to unravel this role of actin in this and other secretory pathways. 


\section{Concluding remarks}

Cholesterol has long been discovered, but its influence in cell membranes and cellular functions are just now being revealed. With the aid of new and more powerful microscopy techniques it was possible to determine its role in actin cytoskeleton organization and cellular mechanics, as well as the consequences of these processes in other cellular functions, such as cell secretion. This has many implications in regulation of different tissue functions. In particular, regulation of lysosome secretion by cholesterol, rafts and actin, may influence the knowledge we have about plasma membrane repair and help understand a lot of diseases related to this organelle. As briefly shown above, cellular processes are all connected and by pulling one string many others might be disturbed. As for cholesterol, many of the roles previously accounted directly to cholesterol and membrane rafts are in fact coordinated by a cascade of other events connected by its interaction and regulation of cell cytoskeleton. Being able to interpret and manipulate this system will certainly contribute to control a lot of cellular functions, especially in pathological conditions.

\section{Acknowledgements}

It was certainly impossible to cover the entire history and even less all the microscopy techniques involved with the study of cholesterol, membrane rafts and their influence on cellular mechanics and cell secretion. Therefore, I would like to acknowledge not only the work shown here but also many others that were not mentioned but were still valuable for the comprehension of this topic. For sure, they have contributed much more to the field than my contribution so far. I also would like to especially thank Dr. Barbara Hissa, Dr. Ubirajara Agero and Dr. Oscar Mesquita, for introducing me to the world of cellular mechanics and for allowing me to use the advantages of defocusing microscopy to unravel the role of cholesterol in regulation of lysosomal secretion. Much has been learned and still a lot is yet to be discovered. And last but not least, I would like to thank to the Brazilian funding agencies, which has allowed me to carry out my research, CAPES, CNPq and FAPEMIG.

\section{References}

[1] U. Agero et al., Cell surface fluctuations studied with defocusing microscopy, Phys Rev E Stat Nonlin Soft Matter Phys 67(5 Pt 1) (2003), 051904. doi:10.1103/PhysRevE.67.051904.

[2] U. Agero et al., Defocusing microscopy, Microsc Res Tech 65(3) (2004), 159-165. doi:10.1002/jemt.20117.

[3] L.O. Andrade and N.W. Andrews, Lysosomal fusion is essential for the retention of Trypanosoma cruzi inside host cells, J Exp Med 200(9) (2004), 1135-1143.

[4] R. Aoki et al., Duration of fusion pore opening and the amount of hormone released are regulated by myosin II during kiss-and-run exocytosis, Biochem J 429(3) (2010), 497-504. doi:10.1042/BJ20091839.

[5] A. Ashkin et al., Observation of a single-beam gradient force optical trap for dielectric particles, Opt Lett 11(5) (1986), 288.

[6] A. Ashkin et al., Force generation of organelle transport measured in vivo by an infrared laser trap, Nature 348(6299) (1990), 346-348. doi:10.1038/348346a0.

[7] A. Ashkin, J.M. Dziedzic and T. Yamane, Optical trapping and manipulation of single cells using infrared laser beams, Nature 330(6150) (1987), 769-771. doi:10.1038/330769a0.

[8] D. Aunis and M.F. Bader, The cytoskeleton as a barrier to exocytosis in secretory cells, J Exp Biol 139 (1988), 253-266.

[9] J.A. Barden et al., Localization of the phalloidin and nucleotide-binding sites on actin, Eur J Biochem 162(3) (1987), 583-588. doi:10.1111/j.1432-1033.1987.tb10679.x.

[10] E.S. Barrias et al., Participation of macrophage membrane rafts in Trypanosoma cruzi invasion process, Biochem Biophys Res Commun 363(3) (2007), 828-834. doi:10.1016/j.bbrc.2007.09.068. 
[11] P. Bhat and P. Thorn, Myosin 2 maintains an open exocytic fusion pore in secretory epithelial cells, Mol Biol Cell 20(6) (2009), 1795-1803.

[12] G. Binnig, C.F. Quate and C. Gerber, Atomic force microscope, Phys Rev Lett 56(9) (1986), 930-933. doi:10.1103/ PhysRevLett.56.930.

[13] G. Binnig and H. Rohrer, Scanning tunneling microscopy, IBM Journal of Research and Development 30(4) (1981), $355-369$.

[14] A. Blair et al., Oxidized low density lipoprotein displaces endothelial nitric-oxide synthase (eNOS) from plasmalemmal caveolae and impairs ENOS activation, J Biol Chem 274(45) (1999), 32512-32519.

[15] S.M. Block, L.S. Goldstein and B.J. Schnapp, Bead movement by single kinesin molecules studied with optical tweezers, Nature 348(6299) (1990), 348-352. doi:10.1038/348348a0.

[16] A. Boerke et al., Capacitation-dependent reorganization of microdomains in the apical sperm head plasma membrane: Functional relationship with zona binding and the zona-induced acrosome reaction, Theriogenology, 70(8) (2008), 11881196.

[17] F. Brochard-Wyart et al., Hydrodynamic narrowing of tubes extruded from cells, Proc Natl Acad Sci U S A 103(20) (2006), 7660-7663. doi:10.1073/pnas.0602012103.

[18] R.E. Brown, Sphingolipid organization in biomembranes: What physical studies of model membranes reveal, $J$ Cell Sci 111(Pt 1) (1998), 1-9.

[19] B.A. Burleigh and N.W. Andrews, A 120-kDa alkaline peptidase from Trypanosoma cruzi is involved in the generation of a novel $\mathrm{Ca}(2+)$-signaling factor for mammalian cells, J Biol Chem, 270(10) (1995), 5172-5180.

[20] F.J. Byfield et al., Cholesterol depletion increases membrane stiffness of aortic endothelial cells, Biophys J 87(5) (2004), 3336-3343. doi:10.1529/biophysj.104.040634.

[21] F.J. Byfield et al., OxLDL increases endothelial stiffness, force generation, and network formation, J Lipid Res 47 (2006), 715-723.

[22] E.V. Caler et al., The exocytosis-regulatory protein synaptotagmin VII mediates cell invasion by Trypanosoma cruzi, J Exp Med 193(9) (2001), 1097-1104.

[23] P. Caroni, New EMBO members' review: actin cytoskeleton regulation through modulation of $\mathrm{PI}(4,5) \mathrm{P}(2) \mathrm{rafts}, E M B O$ $J$ 20(16) (2001), 4332-4336.

[24] L.H. Chamberlain, R.D. Burgoyne and G.W. Gould, SNARE proteins are highly enriched in lipid rafts in PC12 cells: Implications for the spatial control of exocytosis, Proc Natl Acad Sci U S A 98(10) (2001), 5619-5624. doi:10.1073/ pnas.091502398.

[25] Y.A. Chen and R.H. Scheller, SNARE-mediated membrane fusion, Nat Rev Mol Cell Biol 2(2) (2001), 98-106. doi:10. $1038 / 35052017$.

[26] M.E. Chevreul, Recherches chimiques sur les corps gras, et particulièrement sur leurs combinaisons avec les alcalis. Sixième mémoire. Examen des graisses d'homme, de mouton, de boeuf, de jaguar et d'oie (Chemical researches on fatty substances, and particularly on their combinations o filippos ine kapios with alkalis. Sixth memoir. Study of human, sheep, beef, jaguar and goose fat), Annales de Chimie et de Physique 2 (1816), 339-372.

[27] G.R. Chichili and W. Rodgers, Cytoskeleton-membrane interactions in membrane raft structure, Cell Mol Life Sci 66(14) (2009), 2319-2328.

[28] J.A. Chouinard et al., Oxidized-LDL induce morphological changes and increase stiffness of endothelial cells, Exp Cell Res, 314 (2008), 3007-3016.

[29] V.I. Chubinskiy-Nadezhdin, Y.A. Negulyaev and E.A. Morachevskaya, Cholesterol depletion-induced inhibition of stretch-activated channels is mediated via actin rearrangement, Biochem Biophys Res Commun 412(1) (2011), 80-85. doi:10.1016/j.bbrc.2011.07.046.

[30] J. Coelho Neto et al., Measuring optical and mechanical properties of a living cell with defocusing microscopy, Biophys J 91(3) (2006), 1108-1115. doi:10.1529/biophysj.105.073783.

[31] A. Dahan et al., The solubility-permeability interplay in using cyclodextrins as pharmaceutical solubilizers: Mechanistic modeling and application to progesterone, J Pharm Sci 99(6) (2010), 2739-2749. doi:10.1002/jps.22033.

[32] J. Dai and M.P. Sheetz, Mechanical properties of neuronal growth cone membranes studied by tether formation with laser optical tweezers, Biophys J 68(3) (1995), 988-996. doi:10.1016/S0006-3495(95)80274-2.

[33] I. Dalle-Donne et al., The actin cytoskeleton response to oxidants: From small heat shock protein phosphorylation to changes in the redox state of actin itself, Free Radic Biol Med 31(12) (2001), 1624-1632.

[34] R.A. Demel and B. De Kruyff, The function of sterols in membranes, Biochim Biophys Acta 457(2) (1976), 109-132. doi:10.1016/0304-4157(76)90008-3.

[35] B.W. Doreian, T.G. Fulop and C.B. Smith, Myosin II activation and actin reorganization regulate the mode of quantal exocytosis in mouse adrenal chromaffin cells, J Neurosci 28(17) (2008), 4470-4478. doi:10.1523/JNEUROSCI.000808.2008.

[36] M.L. Dorta et al., Ca2+ signal induced by Trypanosoma cruzi metacyclic trypomastigote surface molecules implicated in mammalian cell invasion, Mol Biochem Parasitol 73(1-2) (1995), 285-289. 
[37] D. Dvornik and P. Hill, Effect of long-term administration of AY-9944, an inhibitor of 7-dehydrocholesterol delta 7reductase, on serum and tissue lipids in the rat, J Lipid Res 9 (1968), 587-595.

[38] M.C. Fernandes et al., Novel strategy in Trypanosoma cruzi cell invasion: Implication of cholesterol and host cell microdomains, Int J Parasitol, 37(13) (2007), 1431-1441.

[39] M.C. Fernandes et al., Trypanosoma cruzi subverts the sphingomyelinase-mediated plasma membrane repair pathway for cell invasion, J Exp Med 208(5) (2011), 909-921. doi:10.1084/jem.20102518.

[40] M.C. Fernandes and N.W. Andrews, Host cell invasion by Trypanosoma cruzi: A unique strategy that promotes persistence, FEMS Microbiol Rev 36(3) (2012), 734-747.

[41] B.M. Gadella et al., Sperm head membrane reorganisation during capacitation, Int J Dev Biol 52(5-6) (2008), 473-480.

[42] N. Gavara, A beginner's guide to atomic force microscopy probing for cell mechanics, Microsc Res Tech (2016).

[43] M.S. Giretti and T. Simoncini, Rapid regulatory actions of sex steroids on cell movement through the actin cytoskeleton, Steroids 73(9-10) (2008), 895-900.

[44] K.H. Guenther, P.G. Wierer and J.M. Bennett, Surface roughness measurements of low-scatter mirrors and roughness standards, Applied Optics 23(21) (1984), 3820-3836. doi:10.1364/AO.23.003820.

[45] S.J. Gunst, D.D. Tang and A. Opazo Saez, Cytoskeletal remodeling of the airway smooth muscle cell: A mechanism for adaptation to mechanical forces in the lung, Respir Physiol Neurobiol 137(2-3) (2003), 151-168.

[46] P.K. Hansma et al., Scanning tunneling microscopy and atomic force microscopy: Application to biology and technology, Science 242(4876) (1988), 209-216. doi:10.1126/science.3051380.

[47] T. Harder et al., Specific release of membrane-bound annexin II and cortical cytoskeletal elements by sequestration of membrane cholesterol, Mol Biol Cell 8(3) (1997), 533-545.

[48] T. Harder and K. Simons, Clusters of glycolipid and glycosylphosphatidylinositol-anchored proteins in lymphoid cells: Accumulation of actin regulated by local tyrosine phosphorylation, Eur J Immunol 29(2) (1999), 556-562. doi:10.1002/ (SICI)1521-4141(199902)29:02<556::AID-IMMU556>3.0.CO;2-2.

[49] T. Hevonoja et al., Structure of low density lipoprotein (LDL) particles: Basis for understanding molecular changes in modified LDL, Biochim Biophys Acta 1488(3) (2000), 189-210.

[50] B. Hissa et al., Membrane cholesterol regulates lysosome-plasma membrane fusion events and modulates Trypanosoma cruzi invasion of host cells. PLoS Negl Trop Dis 6(3) (2012), e1583.

[51] B. Hissa et al., Membrane cholesterol removal changes mechanical properties of cells and induces secretion of a specific pool of lysosomes, PLoS One 8(12) (2013), e82988.

[52] B. Hissa and L.O. Andrade, Trypasonoma cruzi uses a specific subset of host cell lysosomes for cell invasion, Parasitol Int 64(2) (2015), 135-138.

[53] F.M. Hochmuth et al., Deformation and flow of membrane into tethers extracted from neuronal growth cones, Biophys $J$ 70(1) (1996), 358-369. doi:10.1016/S0006-3495(96)79577-2.

[54] Y.U. Katagiri, N. Kiyokawa and J. Fujimoto, A role for lipid rafts in immune cell signaling, Microbiol Immunol, 45(1) (2001), 1-8.

[55] S. Kawato, K. Kinosita Jr. and A. Ikegami, Effect of cholesterol on the molecular motion in the hydrocarbon region of lecithin bilayers studied by nanosecond fluorescence techniques, Biochemistry 17(23) (1978), 5026-5031.

[56] N. Khatibzadeh et al., Effects of cholesterol on nano-mechanical properties of the living cell plasma membrane, Soft Matter 8(32) (2012), 8350-8360. doi:10.1039/c2sm25263e.

[57] N. Khatibzadeh et al., Effects of plasma membrane cholesterol level and cytoskeleton F-actin on cell protrusion mechanics, PLoS One 8(2) (2013), e57147.

[58] E.P. Kilsdonk et al., Cellular cholesterol efflux mediated by cyclodextrins, J Biol Chem 270 (1995), 17250-17256.

[59] T.K. Klausen et al., Cholesterol modulates the volume-regulated anion current in Ehrlich-Lettre ascites cells via effects on Rho and F-actin, Am J Physiol Cell Physiol 291(4) (2006), C757-C771. doi:10.1152/ajpcell.00029.2006.

[60] G.B. Kowalsky, F.J. Byfield and I. Levitan, oxLDL facilitates flow-induced realignment of aortic endothelial cells, $A m J$ Physiol Cell Physiol 295(2) (2008), C332-C340. doi:10.1152/ajpcell.00335.2007.

[61] H.S. Kruth and M. Vaughan, Quantification of low density lipoprotein binding and cholesterol accumulation by single human fibroblasts using fluorescence microscopy, J Lipid Res 21(1) (1980), 123-130.

[62] S.V. Kurkov and T. Loftsson Cyclodextrins, Int J Pharm 453(1) (2013), 167-180.

[63] J. Kwik et al., Membrane cholesterol, lateral mobility, and the phosphatidylinositol 4, 5-bisphosphate-dependent organization of cell actin, Proc Natl Acad Sci U S A 100(24) (2003), 13964-13969. doi:10.1073/pnas.2336102100.

[64] T. Lang et al., SNAREs are concentrated in cholesterol-dependent clusters that define docking and fusion sites for exocytosis, Embo J 20(9) (2001), 2202-2213. doi:10.1093/emboj/20.9.2202.

[65] Y. Lange et al., Plasma membranes contain half the phospholipid and $90 \%$ of the cholesterol and sphingomyelin in cultured human fibroblasts, J Biol Chem 264 (1989), 3786-3793.

[66] H.Q. Le et al., Mechanical regulation of transcription controls polycomb-mediated gene silencing during lineage commitment, Nat Cell Biol 18(8) (2016), 864-875. doi:10.1038/ncb3387.

[67] A.M. Lengsfeld et al., Interaction of phalloidin with actin, Proc Natl Acad Sci U S A 71(7) (1974), 2803-2807. 
[68] I. Levitan and K.J. Gooch, Lipid rafts in membrane-cytoskeleton interactions and control of cellular biomechanics: Actions of oxLDL, Antioxid Redox Signal, 9 (2007), 1519-1534.

[69] D. Lichtenberg, F.M. Goni and H. Heerklotz, Detergent-resistant membranes should not be identified with membrane rafts, Trends Biochem Sci 30(8) (2005), 430-436. doi:10.1016/j.tibs.2005.06.004.

[70] S.H. Low et al., Syntaxins 3 and 4 are concentrated in separate clusters on the plasma membrane before the establishment of cell polarity, Mol Biol Cell 17(2) (2006), 977-989.

[71] A. Masedunskas et al., Role for the actomyosin complex in regulated exocytosis revealed by intravital microscopy, Proc Natl Acad Sci U S A 108(33) (2011), 13552-13557. doi:10.1073/pnas.1016778108.

[72] P. Miklavc et al., Ca2+-dependent actin coating of lamellar bodies after exocytotic fusion: A prerequisite for content release or kiss-and-run, Ann N Y Acad Sci 1152 (2009), 43-52.

[73] M. Moran and M.C. Miceli, Engagement of GPI-linked CD48 contributes to TCR signals and cytoskeletal reorganization: A role for lipid rafts in T cell activation, Immunity 9(6) (1998), 787-796. doi:10.1016/S1074-7613(00)80644-5.

[74] S. Muallem et al., Actin filament disassembly is a sufficient final trigger for exocytosis in nonexcitable cells, $J$ Cell Biol 128(4) (1995), 589-598.

[75] T. Nebl et al., Proteomic analysis of a detergent-resistant membrane skeleton from neutrophil plasma membranes, $J$ Biol Chem 277(45) (2002), 43399-43409.

[76] T. Nemoto et al., Stabilization of exocytosis by dynamic F-actin coating of zymogen granules in pancreatic acini, $J$ Biol Chem 279(36) (2004), 37544-37550.

[77] C.M. Nightingale et al., Patterns of body size and adiposity among UK children of South Asian, black African-Caribbean and white European origin: Child Heart and Health Study in England (CHASE study), Int J Epidemiol 40(1) (2011), 3344

[78] L.L. Norman et al., Modification of cellular cholesterol content affects traction force, adhesion and cell spreading, Cell Mol Bioeng 3(2) (2010), 151-162. doi:10.1007/s12195-010-0119-x.

[79] M. Ohara-Imaizumi et al., TIRF imaging of docking and fusion of single insulin granule motion in primary rat pancreatic beta-cells: Different behaviour of granule motion between normal and Goto-Kakizaki diabetic rat beta-cells, Biochem J 381(Pt 1) (2004), 13-18.

[80] Y. Ohtani et al., Differential effects of alpha-, beta- and gamma-cyclodextrins on human erythrocytes, Eur J Biochem 186(1-2) (1989), 17-22.

[81] S. Parthasarathy et al., Oxidized low-density lipoprotein, Methods Mol Biol 610 (2010), 403-417. doi:10.1007/978-160327-029-8_24.

[82] A. Pendleton and A. Koffer, Effects of latrunculin reveal requirements for the actin cytoskeleton during secretion from mast cells, Cell Motil Cytoskeleton, 48(1) (2001), 37-51.

[83] L.J. Pike, Rafts defined: A report on the Keystone Symposium on Lipid Rafts and Cell Function, J Lipid Res 47(7) (2006), 1597-1598. doi:10.1194/jlr.E600002-JLR200.

[84] J. Pitha et al., Drug solubilizers to aid pharmacologists: Amorphous cyclodextrin derivatives, Life Sci 43(6) (1988), 493-502. doi:10.1016/0024-3205(88)90150-6.

[85] A. Pralle et al., Sphingolipid-cholesterol rafts diffuse as small entities in the plasma membrane of mammalian cells, J Cell Biol 148(5) (2000), 997-1008.

[86] N. Puri and P.A. Roche, Ternary SNARE complexes are enriched in lipid rafts during mast cell exocytosis, Traffic 7(11) (2006), 1482-1494.

[87] M. Qi et al., Cholesterol-regulated stress fiber formation, J Cell Biochem 106(6) (2009), 1031-1040.

[88] B. Ramstedt and J.P. Slotte, Membrane properties of sphingomyelins, FEBS Lett 531 (2002), 33-37.

[89] S.K. Rao et al., Identification of SNAREs involved in synaptotagmin VII-regulated lysosomal exocytosis, J Biol Chem 279(19) (2004), 20471-20479.

[90] A. Reddy, E.V. Caler and N.W. Andrews, Plasma membrane repair is mediated by Ca(2+)-regulated exocytosis of lysosomes, Cell 106(2) (2001), 157-169.

[91] J.R. Silvius, Role of cholesterol in lipid raft formation: Lessons from lipid model systems, Biochim Biophys Acta 1610(2) (2003), 174-183.

[92] K. Simons and E. Ikonen, Functional rafts in cell membranes, Nature 387 (1997), 569-572.

[93] K. Simons and D. Toomre, Lipid rafts and signal transduction, Nat Rev Mol Cell Biol 1(1) (2000), 31-39. doi:10.1038/ 35036052 .

[94] K. Simons and W.L. Vaz, Model systems, lipid rafts, and cell membranes, Annu Rev Biophys Biomol Struct 33 (2004), 269-295.

[95] M. Stehr et al., Platelet derived growth factor-BB is a potent mitogen for rat ureteral and human bladder smooth muscle cells: Dependence on lipid rafts for cell signaling, J Urol 169(3) (2003), 1165-1170.

[96] C.D. Stubbs and A.D. Smith, The modification of mammalian membrane polyunsaturated fatty acid composition in relation to membrane fluidity and function, Biochim Biophys Acta 779(1) (1984), 89-137. doi:10.1016/03044157(84)90005-4. 
[97] M. Sun et al., The effect of cellular cholesterol on membrane-cytoskeleton adhesion, J Cell Sci 120(Pt 13) (2007), 2223-2231. doi:10.1242/jcs.001370.

[98] K. Svoboda and S.M. Block, Force and velocity measured for single kinesin molecules, Cell 77(5) (1994), $773-784$.

[99] C. Tam et al., Exocytosis of acid sphingomyelinase by wounded cells promotes endocytosis and plasma membrane repair, J Cell Biol 189(6) (2010), 1027-1038. doi:10.1083/jcb.201003053.

[100] I. Tardieux et al., Lysosome recruitment and fusion are early events required for trypanosome invasion of mammalian cells, Cell 71(7) (1992), 1117-1130.

[101] I. Tardieux, M.H. Nathanson and N.W. Andrews, Role in host cell invasion of Trypanosoma cruzi-induced cytosolic-free Ca2+ transients, J Exp Med 179(3) (1994), 1017-1022.

[102] P.S. Tsai et al., Syntaxin and VAMP association with lipid rafts depends on cholesterol depletion in capacitating sperm cells, Mol Membr Biol 24(4) (2007), 313-324.

[103] M.R. Turvey and P. Thorn, Lysine-fixable dye tracing of exocytosis shows F-actin coating is a step that follows granule fusion in pancreatic acinar cells, Pflugers Arch 448(5) (2004), 552-555. doi:10.1007/s00424-004-1288-z.

[104] W.J. Van Blitterswijk, R.P. Van Hoeven and B.W. Van der Meer, Lipid structural order parameters (reciprocal of fluidity) in biomembranes derived from steady-state fluorescence polarization measurements, Biochim Biophys Acta 644(2) (1981), 323-332. doi:10.1016/0005-2736(81)90390-4.

[105] F. Van Laethem and O. Leo, Membrane lipid rafts: New targets for immunoregulation, Curr Mol Med 2(6) (2002), $557-570$.

[106] M. Versaevel, T. Grevesse and S. Gabriele, Spatial coordination between cell and nuclear shape within micropatterned endothelial cells, Nat Commun 3 (2012), 671.

[107] S.E. Wilkowsky et al., Trypanosoma cruzi: Phosphatidylinositol 3-kinase and protein kinase B activation is associated with parasite invasion, Exp Cell Res 264(2) (2001), 211-218.

[108] E. Wulf et al., Fluorescent phallotoxin, a tool for the visualization of cellular actin, Proc Natl Acad Sci U S A 76(9) (1979), 4498-4502. doi:10.1073/pnas.76.9.4498.

[109] J. Xu et al., Mechanism of polarized lysosome exocytosis in epithelial cells, J Cell Sci 125(Pt 24) (2012), 5937-5943. doi: $10.1242 /$ jcs. 109421.

[110] P.G. Yancey et al., Cellular cholesterol efflux mediated by cyclodextrins. Demonstration of kinetic pools and mechanism of efflux, J Biol Chem 271(27) (1996), 16026-16034.

[111] P.L. Yeagle, Cholesterol and the cell membrane, Biochim Biophys Acta 822(3-4) (1985), 267-287.

[112] H.L. Yin and P.A. Janmey, Phosphoinositide regulation of the actin cytoskeleton, Annu Rev Physiol 65 (2003), 761-789. doi:10.1146/annurev.physiol.65.092101.142517.

[113] J. Yu, D.A. Fischman and T.L. Steck, Selective solubilization of proteins and phospholipids from red blood cell membranes by nonionic detergents, J Supramol Struct 1(3) (1973), 233-248.

[114] O. Zamir and M.P. Charlton, Cholesterol and synaptic transmitter release at crayfish neuromuscular junctions, J Physiol 571(Pt 1) (2006), 83-99. doi:10.1113/jphysiol.2005.098319. 